\title{
Pomona Large Vessel Occlusion Screening Tool for Prehospital and Emergency Room Settings
}

\author{
Kessarin Panichpisal ${ }^{a}$ Kenneth Nugent ${ }^{b}$ Maharaj Singh ${ }^{c}$ \\ Richard Rovin $^{a}$ Reji Babygirija ${ }^{c}$ Yogesh Moradiya ${ }^{d}$ Karen Tse-Chang ${ }^{e}$ \\ Kimberly A. Jones ${ }^{e}$ Katrina J. Woolfolk ${ }^{e}$ Debbie Keasler ${ }^{\mathrm{e}}$ \\ Bhupat Desai $^{\text {e }}$ Parinda Sakdanaraseth ${ }^{f}$ Paphavee Sakdanaraseth 9 \\ Alimohammad Moalem ${ }^{\text {h }}$ Nazli Janjua ${ }^{\mathrm{e}}{ }^{\mathrm{i}}$

\begin{abstract}
${ }^{a}$ Aurora Neuroscience Innovation Institute, Aurora St. Luke's Medical Center, Milwaukee, WI, USA; ${ }^{b}$ Department of Internal Medicine, Texas Tech University Health Sciences Center, Lubbock, TX, USA; ' Aurora Research Institute, Aurora Sinai Medical Center, Milwaukee, WI, USA; d Department of Neurosurgery, Baptist Medical Center, Jacksonville, FL, USA; e Pomona Valley Hospital, Pomona, CA, USA; ${ }^{f}$ Department of Creative Arts, Faculty of Fine and Applied Arts, Chulalongkorn University, Bangkok, Thailand; 9 Department of Industrial Design, Faculty of Architecture, Chulalongkorn University, Bangkok, Thailand; h Department of Surgery, Kaiser Permanente Los Angeles Medical Center, Los Angeles, CA, USA; 'Asia Pacific Comprehensive Stroke Institute, Pomona, CA, USA
\end{abstract}

\section{Keywords}

Acute stroke $\cdot$ Prehospital emergency care $\cdot$ Vessel occlusion $\cdot$ Scales

\begin{abstract}
Background: Early identification of patients with acute ischemic strokes due to large vessel occlusions (LVO) is critical. We propose a simple risk score model to predict LVO. Method: The proposed scale (Pomona Scale) ranges from 0 to 3 and includes 3 items: gaze deviation, expressive aphasia, and neglect. We reviewed a cohort of all acute stroke activation patients between February 2014 and January 2016. The predictive performance of the Pomona Scale was determined and compared with several National Institutes of Health Stroke Scale (NIHSS) cutoffs ( $\geq 4, \geq 6, \geq 8$, and $\geq 10$ ), the Los Angeles Motor Scale (LAMS), the Cincinnati Prehospital Stroke Severity (CPSS) scale, the Vision Aphasia and Neglect Scale (VAN), and the Prehospital Acute Stroke Severity Scale (PASS). Results: LVO was detected in 94 of 776 acute stroke activations (12\%). A Pomona Scale $\geq 2$ had comparable accuracy to predict LVO as the VAN and CPSS scales and higher accuracy than Pomona Scale $\geq 1$, LAMS, PASS, and NIHSS. A Pomona Scale $\geq 2$ had an accuracy (area under the curve) of 0.79 , a sensitivity of 0.86 , a specificity of
\end{abstract}


0.70 , a positive predictive value of 0.71 , and a negative predictive value of 0.97 for the detection of LVO. We also found that the presence of either neglect or gaze deviation alone had comparable accuracy of 0.79 as Pomona Scale $\geq 2$ to detect LVO. Conclusion: The Pomona Scale is a simple and accurate scale to predict LVO. In addition, the presence of either gaze deviation or neglect also suggests the possibility of LVO.

(c) 2018 S. Karger AG, Base

\section{Background}

Each year in the United States approximately 692,000 people have new or recurrent ischemic strokes [1]. In $40-50 \%$ of these patients, this is due to large vessel occlusions (LVO) [2]. A recent population-based study reported that the estimated incidence of acute LVO was 77,569 per year in the United States, and that fewer than 11,000 thrombectomies were performed in 2015 [3]. Thus, the majority of patients with LVO stroke do not receive potentially beneficial interventions. The principal reason is delayed diagnosis and subsequent delayed transfer to an endovascular capable stroke center [4]. Therefore, early detection of patients with LVO is important. At present, there are several scales that use different clinical signs for identifying LVO patients; however, these scales are not widely used in clinical practice [5]. The most popular scales/scores are the Los Angeles Motor Scale (LAMS), the Cincinnati Prehospital Stroke Severity (CPSS) scale, the Rapid Arterial Occlusion Evaluation (RACE) scale, and the Vision Aphasia and Neglect Scale (VAN) [6-9]. We propose a simple screening tool that is easy to use in both prehospital and emergency room settings (the Pomona Scale).

\section{Methods}

The Pomona Valley Hospital Institutional Review Board considered this a retrospective chart review research study. All charts for patients presenting with acute stroke alert activation at Pomona Valley Hospital (a certified primary stroke center with endovascular capability in Pomona, CA, USA) between February 2014 and January 2016 were reviewed. Demographic data were collected.

LVO was determined by computed tomography (CT) angiogram, magnetic resonance angiogram, or cerebral angiogram and involved the distal internal carotid artery, middle cerebral artery (M1), or basilar artery. We excluded distal middle cerebral artery branch (M2, M3, and M4), anterior cerebral artery, posterior cerebral artery, and vertebral artery occlusions because the standard of care using thrombectomy for these LVO is less rigidly established [10].

We also excluded patients who had a final diagnosis of stroke but no vascular imaging. At our hospital, noncontrast CT head scans are obtained followed by CT angiograms (if no contraindication) for all patients with suspected acute ischemic stroke who were last known to be well within $8 \mathrm{~h}$ of presentation and who had an initial National Institutes of Health Stroke Scale (NIHSS) score $\geq 4$ or $<4$ if the on-call neurologist suspected LVO. The NIHSS was routinely assessed by certified NIHSS nurses in all acute stroke activations. For wake-up stroke patients, triage for revascularization treatment was per clinician discretion. We reviewed all vascular imaging for all acute stroke patients, including digital subtraction angiography, CT angiography, and/or magnetic resonance angiography.

Each chart was further reviewed for the clinical features that comprised the elements of the various scales. We collected the total NIHSS score and specific NIHSS data, including level of consciousness (1a), LOC (level of consciousness) question (1b), LOC command (1c), best gaze, visual field, facial weakness, motor arm weakness, aphasia, and neglect. Expressive aphasia is not routinely identified in the aphasia score in the NIHSS. Therefore, for a patient with a positive score for aphasia, we reviewed physician notes to determine whether or not the patient had expressive aphasia. 
Our new Pomona Scale ranges from 0 to 3 and includes the following signs: gaze deviation, expressive aphasia, and neglect, each given 1 point when present. We hypothesized that having Pomona Scale $\geq 2$ would have a high sensitivity, specificity, and accuracy to predict LVO. The LAMS is a graded 3-item scale: facial droop (absent $=0$, present $=1$ ), arm drift (absent $=0$, drifts $=1$, falls rapidly $=2$ ), and grip strength (normal $=0$, weak $=1$, none $=2$ ) with a range from 0 to 5 [6]. The CPSS scale grades 3 NIHSS items: 2 points for the presence of conjugate gaze, 1 point for the presence of arm weakness, and 1 point for altered level of consciousness with a range of 0-4 [8]. The VAN assesses upper extremity drift (weakness), vision problem, aphasia, and neglect [9]. The score is either positive or negative; a positive score means the patient has weakness and 1 or more of the other findings. The Prehospital Acute Stroke Severity Scale (PASS) also grades 3 NIHSS items (gaze deviation, arm weakness, and level of consciousness, absent $=0$, present $=1$ ) with a range of 0-3 [5]. For purposes of comparison, the scales were considered positive for LVO if the Pomona Scale was $\geq 2$, the LAMS $\geq 4$, the CPSS scale $\geq 2$, the VAN positive, and the PASS $\geq 2$.

We calculated each scale's sensitivity, specificity, positive and negative likelihood ratios, positive and negative predictive values, and performance using receiver operating characteristic curve analysis (area under the receiver operating characteristic curve [AUC]). A 2-sided $p$ value of $<0.05$ was considered significant. All statistical analysis was performed using SAS version 9.4, SAS Institute, Cary, NC, USA.

\section{Results}

Seven hundred ninety-one patients triggered an acute stroke activation; 15 patients that did not receive vascular imaging due to exclusion criteria were not included in the analysis. Four hundred and forty-four (57\%) patients were female. The median age was 71 years (interquartile range [IQR] 59-84). There were 94 patients with acute ischemic strokes due to LVO: 48 patients with M1 occlusions, 41 with internal carotid artery occlusions, and 5 with basilar artery occlusions. There were 38 patients with distal artery occlusion (M2 and M3 branches). Of 30 stroke patients with M2 branch occlusion, 24 (80\%) had Pomona $\geq 2$. Of 8 stroke patients with M3 branch occlusion, 3 (38\%) had Pomona $\geq 2$.

Among the LVO patients, left hemispheric involvement was present in 30 patients and right hemispheric involvement in 59 patients. The 682 acute stroke activations with vascular imaging but no LVO included 286 acute ischemic strokes, 110 transient ischemic attacks, 93 intracranial hemorrhages, and 193 stroke mimics. Stroke mimic patients had metabolic disturbances $(n=52)$, seizures $(n=43)$, psychiatric diagnoses $(n=20)$, migraine headaches $(n=13)$, and other conditions $(n=105)$. The median NIHSS score in patients with acute ischemic strokes with LVO was 20 (IQR 17-26) compared to 8 (IQR 4-17) in patients without LVO (Table 1).

Using NIHSS items, the most accurate predictors of LVO were gaze deviation, neglect, and visual field defect with AUCs of 0.79, 0.79, and 0.82, respectively (Table 2). We found that visual field defects always presented with either gaze deviation or neglect in LVO patients. A Pomona Scale $\geq 2$ had comparable accuracy to predict LVO as the VAN and CPSS scales and a higher accuracy than Pomona Scale $\geq 1$, LAMS, PASS, and NIHSS (AUC): Pomona $\geq 2=0.79$ as reference; VAN $=0.75, p=0.07$; CPSS $=0.78, p=0.47$; Pomona $\geq 1=0.74, p=0.0067$; LAMS $=$ $0.75, p=0.044$; PASS $=0.76, p=0.046$; NIHSS $\geq 4, p<0.001$; NIHSS $\geq 6, p<0.001$; NIHSS $\geq 8$, $p<0.001$, and NIHSS $\geq 10, p=0.042$; Fig. 1 ). A Pomona Scale $\geq 2$ had a sensitivity of 0.86 , a specificity of 0.71 , a positive predictive value (PPV) of 0.71 , and a negative predictive value (NPV) of 0.97 compared to the VAN $(0.95,0.56,0.23$, and 0.99 , respectively) and the CPSS scale $(0.92,0.81,0.40$, and 0.99 , respectively). A Pomona Scale $\geq 1$ had a sensitivity of 0.98 , a specificity of 0.50 , a PPV of 0.21 , and an NPV of 0.99 (Table 2; Fig. 1).

We unexpectedly found that the presence of either neglect or gaze deviation has comparable accuracy (AUC $=0.79$ ) to predict $L V O$ as Pomona Scale $\geq 2$ with a sensitivity of 0.95 , a specificity of 0.64 , a PPV of 0.27 , and an NPV of 0.99 (Table 3). 
Interventional

Neurology

Table 1. Demographic data

\begin{tabular}{l|l}
\hline Intervent Neurol 2018;7:196-203 \\
\hline DOI: 10.1159/000486515 & $\begin{array}{l}\text { (c) 2018 S. Karger AG, Basel } \\
\text { www.karger.com/ine }\end{array}$ \\
\hline
\end{tabular}

Panichpisal et al.: Pomona Large Vessel Occlusion Screening Tool for Prehospital and Emergency Room Settings

\begin{tabular}{llc}
\hline & LVO & No LVO \\
\hline Subjects & $94(12)$ & $682(88)$ \\
Female & $57(61)$ & $387(57)$ \\
Age, years & $73(64-84)$ & $70(58-83)$ \\
Left hemisphere & $35(37)$ & N/A \\
NIHSS & $20(17-26)$ & $8(4-7)$ \\
Vessel occlusion & & N/A \\
$\quad$ ICA & $41(44)$ & \\
$\quad$ M1 & $48(50)$ & \\
$\quad$ BA & $5(5)$ & \\
\hline AIS & $94(100)$ & $286(42)$ \\
TIA & N/A & $110(16)$ \\
ICH & N/A & $93(14)$ \\
Stroke mimics & N/A & $193(28)$ \\
$\quad$ Metabolic & & $52(27)$ \\
$\quad$ Seizure & & $43(22)$ \\
Psychiatric & & $20(10)$ \\
Migraine & & $13(7)$ \\
Other & & $105(54)$ \\
\hline
\end{tabular}

Values are $n(\%)$ or medians (interquartile ranges). LVO, large vessel occlusion; NIHSS, National Institutes of Health Stroke Scale; ICA, internal carotid artery; BA, basilar artery; AIS, acute ischemic stroke; TIA, transient ischemic attack; ICH, intracerebral hemorrhage; N/A, not applicable.
Fig. 1. Receiver operating characteristic curves comparing the discrimination of Pomona Scale $\geq 2$, Pomona Scale $\geq 1$, neglect/gaze (gaze deviation), VAN, LAMS, CPSS, PASS, and NIHSS for the detection of large vessel occlusion strokes. VAN, Vision Aphasia and Neglect Scale; LAM, Los Angeles Motor Scale; CPSS, Cincinnati Prehospital Stroke Severity Scale; PASS, Prehospital Acute Stroke Severity Scale; NIHSS, National Institutes of Health Stroke Scale. 
Table 2. Performance of NIHSS items for LVO prediction

\begin{tabular}{llllll}
\hline & Sensitivity & Specificity & PPV & NPV & AUC \\
\hline LOC (1a) & $0.23(0.15-0.33)$ & $0.81(0.77-0.83)$ & $0.14(0.10-0.20)$ & $0.88(0.87-0.90)$ & $0.52(0.47-0.57)$ \\
LOC (1b) & $0.60(0.52-0.60)$ & $0.56(0.52-0.60)$ & $0.16(0.13-0.18)$ & $0.91(0.89-0.93)$ & $0.58(0.52-0.63)$ \\
LOC (1c) & $0.49(0.38-0.59)$ & $0.68(0.65-0.72)$ & $0.17(0.14-0.21)$ & $0.91(0.89-0.92)$ & $0.59(0.53-0.64)$ \\
Facial weakness & $0.93(0.85-0.97)$ & $0.50(0.47-0.54)$ & $0.20(0.19-0.22)$ & $0.98(0.96-0.99)$ & $0.72(0.68-0.75)$ \\
Motor (arm) & $0.96(0.89-0.99)$ & $0.41(0.37-0.45)$ & $0.18(0.17-0.19)$ & $0.99(0.96-0.99)$ & $0.68(0.65-0.71)$ \\
Visual field defect & $0.88(0.80-0.94)$ & $0.75(0.72-0.78)$ & $0.33(0.30-0.36)$ & $0.98(0.96-0.99)$ & $0.82(0.78-0.85)$ \\
Gaze deviation & $0.84(0.75-0.91)$ & $0.73(0.70-0.78)$ & $0.30(0.27-0.34)$ & $0.97(0.95-0.98)$ & $0.79(0.75-0.83)$ \\
Expressive aphasia & $0.39(0.29-0.50)$ & $0.64(0.61-0.68)$ & $0.13(0.10-0.17)$ & $0.88(0.87-0.90)$ & $0.52(0.47-0.57)$ \\
Neglect & $0.88(0.80-0.94)$ & $0.69(0.66-0.73)$ & $0.28(0.26-0.31)$ & $0.98(0.96-0.99)$ & $0.79(0.75-0.83)$ \\
\hline
\end{tabular}

Values are scores with 95\% confidence intervals in parentheses; NIHSS $>0$ in listed items. NIHSS, National Institutes of Health Stroke Scale; LVO, large vessel occlusion; PPV, positive predictive value; NPV, negative predictive value; AUC, area under the curve; LOC, level of consciousness.

Table 3. Accuracy of LVO screening scales and different NIHSS cutoff scores in comparison with the Pomona Scale in all subjects $(n=776)$

\begin{tabular}{|c|c|c|c|c|c|c|c|}
\hline & Sensitivity & Specificity & PPV & NPV & $\mathrm{AUC}^{\mathrm{a}}$ & SE & $p$ value \\
\hline Pomona $\geq 2$ & $0.86(0.77-0.92)$ & $0.71(0.67-0.75)$ & $0.71(0.67-0.75)$ & $0.97(0.96-0.98)$ & $0.79(0.75-0.83)$ & 0.020 & reference \\
\hline Pomona $\geq 1$ & $0.98(0.93-1.00)$ & $0.50(0.46-0.54)$ & $0.21(0.20-0.23)$ & $0.99(0.98-1.00)$ & $0.74(0.72-0.76)$ & 0.012 & 0.007 \\
\hline \multicolumn{8}{|l|}{ Neglect/gaze } \\
\hline deviation & $0.95(0.88-0.98)$ & $0.64(0.61-0.68)$ & $0.27(0.25-0.29)$ & $0.99(0.97-1.00)$ & $0.79(0.77-0.82)$ & 0.015 & 0.810 \\
\hline VAN & $0.95(0.88-0.98)$ & $0.56(0.52-0.60)$ & $0.23(0.21-0.25)$ & $0.99(0.97-1.00)$ & $0.77(0.74-0.78)$ & 0.015 & 0.066 \\
\hline LAMS $\geq 4$ & $0.76(0.66-0.84)$ & $0.74(0.70-0.77)$ & $0.28(0.25-0.32)$ & $0.96(0.94-0.97)$ & $0.75(0.72-0.78)$ & 0.024 & 0.044 \\
\hline CPSS $\geq 2$ & $0.92(0.84-0.96)$ & $0.81(0.78-0.84)$ & $0.40(0.36-0.44)$ & $0.99(0.97-0.99)$ & $0.78(0.75-0.82)$ & 0.018 & 0.470 \\
\hline PASS $\geq 2$ & $0.91(0.84-0.96)$ & $0.60(0.57-0.64)$ & $0.24(0.22-0.26)$ & $0.98(0.96-0.99)$ & $0.76(0.73-0.79)$ & 0.017 & 0.046 \\
\hline NIHSS $\geq 4$ & $0.99(0.94-1.00)$ & $0.24(0.21-0.28)$ & $0.15(0.15-0.16)$ & $0.99(0.96-1.00)$ & $0.62(0.60-0.63)$ & 0.010 & $<0.001$ \\
\hline NIHSS $\geq 6$ & $0.99(0.94-1.00)$ & $0.39(0.35-0.42)$ & $0.18(0.17-0.19)$ & $1.00(0.97-1.00)$ & $0.68(0.65-0.70)$ & 0.013 & $<0.001$ \\
\hline NIHSS $\geq 8$ & $0.98(0.93-1.00)$ & $0.48(0.44-0.52)$ & $0.21(0.19-0.22)$ & $0.99(0.98-1.00)$ & $0.71(0.68-0.74)$ & 0.016 & $<0.001$ \\
\hline NIHSS $\geq 10$ & $0.98(0.93-1.00)$ & $0.56(0.52-0.59)$ & $0.23(0.22-0.25)$ & $0.99(0.98-1.00)$ & $0.75(0.72-0.78)$ & 0.016 & 0.042 \\
\hline
\end{tabular}

Values are scores with 95\% confidence intervals in parentheses. LVO, large vessel occlusion; NIHSS, National Institutes of Health Stroke Scale; PPV, positive predictive value; NPV, negative predictive value; AUC, area under the curve; VAN, Vision Aphasia and Neglect Scale; LAMS, Los Angeles Motor Scale; CPSS, Cincinnati Prehospital Stroke Severity Scale; PASS, Prehospital Acute Stroke Severity Scale. ${ }^{\text {a }}$ Accuracy at cut point.

\section{Discussion}

For patients with acute ischemic stroke due to LVO, faster times from symptom onset and imaging to reperfusion are associated with more favorable outcomes [11]. Every 30 min delay from onset to angiographic reperfusion reduces the relative likelihood of a good clinical outcome by $12 \%$ [12]. There is an average 2-h delay in transferring patients to hospitals that have endovascular capability ("drip and ship" approach) $[13,14]$. Routing patients with LVO to comprehensive stroke centers or endovascular capable stroke centers directly ("mothership" approach) is likely to be faster and have higher "good" outcomes for patients, but this remains to be proven [15]. Therefore, early identification of acute ischemic stroke patients with LVO is critical. The optimal LVO screening scale is one that is simple, accurate, reliable, valid, and proven to improve patient outcome [16]. Moreover, the screening tool should be applicable in the field so that paramedics can use it for pre-arrival notification and patient triage and in the emergency rooms without acute vascular imaging availability $[15,16]$. 
Our study showed that the Pomona Scale $\geq 2$ or the presence of either neglect or gaze deviation had high sensitivity and a moderate specificity to identify LVO patients. It performed better than more complex scales. The Pomona Scale is very simple, scoring just 3 components of the routine neurological examination: neglect, expressive aphasia, and gaze deviation.

Criteria used to select exam elements for the Pomona Scale included relative ease of testing and ability to differentiate LVO from non-LVO. We intentionally did not include a visual field test in the Pomona Scale because it is complex to perform. And although motor symptoms strongly correlate with higher NIHSS scores, they are not good discriminators of non-LVO from LVO stroke [4]. Facial weakness and arm weakness are not specific for cortical LVO stroke as they can also occur in subcortical or lacunar strokes. On this basis, we did not include a motor component for our scale.

Several screening tools for LVO have been proposed and evaluated [4-9, 14, 17]. The LAMS includes facial and arm motor weakness, does not evaluate cortical signs, and has low sensitivity [6]. The CPSS scale is a simple scale but does not evaluate aphasia or neglect, which are important cortical signs [8]. The VAN is a very sensitive scale and has an easy acronym. However, it is more complex and has several items to evaluate, including visual field testing (which requires training) [9]. The PASS is derived from a large national ischemic stroke cohort. It is quite simple and highly specific. However, data for the specific location of large artery occlusions are absent, making the interpretation of this scale's performance difficult [5]. The RACE scale has been validated in the prehospital setting, but most patients with LVO in this study were diagnosed with transcranial Doppler. The RACE scale is very complex and has 6 items to evaluate, which is too cumbersome for use in the field. Some of the RACE items' scorings are different from NIHSS scoring, which makes it harder for nurses in the emergency room setting who routinely use the NIHSS [7]. Moreover, the RACE scale had an $80 \%$ falsepositive rate in detecting LVO, but its use significantly decreased door to CT, door to needle, door to groin, and door to recanalization times [18]. Although a high false-positive rate presents significant clinical problems, we think that using a screening tool is better than no tool to guide emergency medical services workers in the triage of patients. The Pomona Scale has a $29 \%$ false-positive rate.

NIHSS scores are complex and impractical to perform in the field. The best cutoff score for the NIHSS to predict LVO is still controversial, although a high NIHSS score is associated with LVO $[19,20]$. Our data showed that different NIHSS cutoffs were not accurate in LVO prediction. One study reported that $13 \%$ of patients with LVO had an NIHSS score of 0 [17]. The sensitivity of these scales ranges from 50 to $64 \%$, which means up to one-third of strokes with LVO would be missed [4-9, 14, 17]. Turc et al. [16] analyzed multiple scales for LVO and found that using published cutoffs to reduce the false-negative rate to $10 \%$ would result in a false-positive rate of 46-100\%. Scheitz et al. [19] also confirmed that using the higher cutoffs of multiple scales will result in an underdiagnosis of LVO and vice versa. Ultimately, due to the therapeutic implications of thrombectomy, trading a higher sensitivity for a lower specificity may be more optimal.

The Field Assessment Stroke Triage for Emergency Destination (FAST-ED) scale was published after we had completed this retrospective review and was not directly applied to our data [4]. The authors reported that the sensitivity, specificity, PPV, NPV, and accuracy of FAST-ED $\geq 4$ were $0.61,0.89,0.72,0.82$, and 0.79 , respectively. The respective values for Pomona $\geq 2$ are $0.86,0.71,0.71,0.97$, and 0.79 , respectively. With a higher sensitivity and lower specificity, the Pomona Scale is more likely to identify patients with LVO. We accept a higher false-positive rate as the morbidity of CT angiogram in non-LVO acute ischemic stroke patients is far less than when missing a patient with LVO. Given the similar accuracy of the 2 scales, ease of use and teachableness become important factors. 
From a neuroanatomical point of view, gaze deviation indicates involvement of either the frontal eye field or paramedian pontine reticular formation, which helps us detect both anterior and posterior LVO [5]. Expressive aphasia indicates the involvement of Broca's area of the dominant hemisphere. Neglect or extinction indicates the involvement of the right temporal lobe, insular lobe, putamen, and caudate nucleus of the nondominant hemisphere [21]. Isolated visual field defects from acute ischemic strokes are very rare (approximately $0.16 \%$ ) [22]. The majority of homonymous visual field defects are caused by occipital lobe involvement (40\%), which is supplied by the posterior cerebral artery [22].

This study has limitations. First, this is a retrospective chart review with a relatively small sample size of LVO compared to other studies. Second, there were $5 \%$ of patients with posterior circulation LVO, making it difficult to evaluate the performance of the scale in these patients. Third, external validation with an independent cohort and prospective evaluation in the prehospital setting are needed. Fourth, clinical signs and symptoms can fluctuate in the first hours following acute ischemic stroke; it is possible that the stroke severity at the time of prehospital examination will change by the time a treatment decision for ischemic stroke is made by medical providers. Finally, we did not test our data with all prehospital screening scales for direct comparison purposes, e.g., RACE and FAST-ED scales. The RACE scale was not utilized due to its complexity and time needed to complete, rendering it less applicable to the in-transit setting, and the FAST-ED scale was published after our retrospective data review was completed. We do not believe that the Pomona Scale will create confusion for emergency medical services providers: it is not designed to discriminate stroke from nonstroke neurological emergency. Rather, it is a complementary tool to further categorize a presumed stroke. It is a simple scale with only 3 clinical elements, each of which only has 2 scores, 0 or 1 for absence or presence. By comparison, the FAST-ED scale has 5 clinical elements, each judged by multiple and somewhat subjective criteria. Our purpose in implementing the Pomona Scale is to easily predict LVO and thereby triage the patients to endovascular capable comprehensive stroke centers, bypassing primary stroke centers.

In conclusion, the Pomona Scale is a simple and accurate screening tool to identify LVO. In addition, the presence of either gaze deviation or neglect also suggests the possibility of LVO. If the Pomona Scale is successfully validated prospectively in an emergency room or prehospital setting, it might be a useful tool for LVO triage.

\section{Disclosure Statement}

The authors have no conflicts of interest to declare.

\section{References}

1 Writing Group Members, Mozaffarian D, Benjamin EJ, Go AS, Arnett DK, Blaha MJ, Cushman M, Das SR, de Ferranti S, Després JP, Fullerton HJ, Howard VJ, Huffman MD, Isasi CR, Jiménez MC, Judd SE, Kissela BM, Lichtman JH, Lisabeth LD, Liu S, Mackey RH, Magid DJ, McGuire DK, Mohler ER 3rd, Moy CS, Muntner P, Mussolino ME, Nasir K, Neumar RW, Nichol G, Palaniappan L, Pandey DK, Reeves MJ, Rodriguez CJ, Rosamond W, Sorlie PD, Stein J, Towfighi A, Turan TN, Virani SS, Woo D, Yeh RW, Turner MB; American Heart Association Statistics Committee; Stroke Statistics Subcommittee: Heart disease and stroke statistics - 2016 update: a report from the American Heart Association. Circulation 2016;133:e38-e360.

2 Rai AT: Red pill, blue pill: reflections on the emerging large vessel stroke "market". J Neurointerv Surg 2015; 7:623-625.

3 Rai AT, Seldon AE, Boo S, Link PS, Domico JR, Tarabishy AR, Lucke-Wold N, Carpenter JS: A population-based incidence of acute large vessel occlusions and thrombectomy eligible patients indicates significant potential for growth of endovascular stroke therapy in the USA. J Neurointerv Surg 2017;9:722-726. 
4 Lima FO, Silva GS, Furie KL, Frankel MR, Lev MH, Camargo EC, Haussen DC, Singhal AB, Koroshetz WJ, Smith WS, Nogueira RG: Field Assessment Stroke Triage for Emergency Destination: a simple and accurate prehospital scale to detect large vessel occlusion strokes. Stroke 2016;47:1997-2002.

5 Hastrup S, Damgaard D, Johnsen SP, Andersen G: Prehospital Acute Stroke Severity Scale to predict large artery occlusion: design and comparison with other scales. Stroke 2016;47:1772-1776.

6 Nazliel B, Starkman S, Liebeskind DS, Ovbiagele B, Kim D, Sanossian N, Ali L, Buck B, Villablanca P, Vinuela F, Duckwiler G, Jahan R, Saver JL: A brief prehospital stroke severity scale identifies ischemic stroke patients harboring persisting large arterial occlusions. Stroke 2008;39:2264-2267.

7 Perez de la Ossa N, Carrera D, Gorchs M, Querol M, Millan M, Gomis M, Dorado L, López-Cancio E, HernándezPérez M, Chicharro V, Escalada X, Jiménez X, Dávalos A: Design and validation of a prehospital stroke scale to predict large arterial occlusion: the Rapid Arterial Occlusion Evaluation Scale. Stroke 2014;45:87-91.

8 Katz BS, McMullan JT, Sucharew H, Adeoye 0, Broderick JP: Design and validation of a prehospital scale to predict stroke severity: Cincinnati Prehospital Stroke Severity Scale. Stroke 2015;46:1508-1512.

9 Teleb MS, Ver Hage A, Carter J, Jayaraman MV, McTaggart RA: Stroke vision, aphasia, neglect (VAN) assessment - a novel emergent large vessel occlusion screening tool: pilot study and comparison with current clinical severity indices. J Neurointerv Surg 2017;9:122-126.

10 Powers WJ, Derdeyn CP, Biller J, Coffey CS, Hoh BL, Jauch EC, Johnston KC, Johnston SC, Khalessi AA, Kidwell CS, Meschia JF, Ovbiagele B, Yavagal DR; American Heart Association Stroke Council: 2015 American Heart Association/American Stroke Association focused update of the 2013 guidelines for the early management of patients with acute ischemic stroke regarding endovascular treatment: a guideline for healthcare professionals from the American Heart Association/American Stroke Association. Stroke 2015;46:3020-3035.

11 Goyal M, Jadhav AP, Bonafe A, Diener H, Mendes Pereira V, Levy E, Baxter B, Jovin T, Jahan R, Menon BK, Saver JL; SWIFT PRIME investigators: Analysis of workflow and time to treatment and the effects on outcome in endovascular treatment of acute ischemic stroke: results from the SWIFT PRIME randomized controlled trial. Radiology 2016;279:888-897.

12 Khatri P, Abruzzo T, Yeatts SD, Nichols C, Broderick JP, Tomsick TA; IMS I and II Investigators: Good clinical outcome after ischemic stroke with successful revascularization is time-dependent. Neurology 2009;73: 1066-1072.

13 Sun CH, Nogueira RG, Glenn BA, Connelly K, Zimmermann S, Anda K, Camp D, Frankel MR, Belagaje SR, Anderson AM, Isakov AP, Gupta R: "Picture to puncture": a novel time metric to enhance outcomes in patients transferred for endovascular reperfusion in acute ischemic stroke. Circulation 2013;127:1139-1148.

14 Singer OC, Dvorak F, du Mesnil de Rochemont R, Lanfermann H, Sitzer M, Neumann-Haefelin T: A simple 3-item stroke scale: comparison with the National Institutes of Health Stroke Scale and prediction of middle cerebral artery occlusion. Stroke 2005;36:773-776.

15 Michel P: Prehospital scales for large vessel occlusion: closing in on a moving target. Stroke 2017;48:247-249.

16 Turc G, Maïer B, Naggara O, Seners P, Isabel C, Tisserand M, Raynouard I, Edjlali M, Calvet D, Baron JC, Mas JL, Oppenheim C: Clinical scales do not reliably identify acute ischemic stroke patients with large-artery occlusion. Stroke 2016;47:1466-1472.

17 Moore RD, Jackson JC, Venkatesh SL, Quarfordt SD, Baxter BW: Revisiting the NIH Stroke Scale as a screening tool for proximal vessel occlusion: can advanced imaging be targeted in acute stroke? J Neurointerv Surg 2016, Epub ahead of print.

18 Zaidi SF, Shawver J, Espinosa Morales A, Salahuddin H, Tietjen G, Lindstrom D, Parquette B, Adams A, Korsnack A, Jumaa MA: Stroke care: initial data from a county-based bypass protocol for patients with acute stroke. J Neurointerv Surg 2017;9:631-635.

19 Scheitz JF, Abdul-Rahim AH, MacIsaac RL, Cooray C, Sucharew H, Kleindorfer D, Khatri P, Broderick JP, Audebert HJ, Ahmed N, Wahlgren N, Endres M, Nolte CH, Lees KR; SITS Scientific Committee: Clinical selection strategies to identify ischemic stroke patients with large anterior vessel occlusion: results from SITS-ISTR (Safe Implementation of Thrombolysis in Stroke International Stroke Thrombolysis Registry). Stroke 2017; 48:290-297.

20 Maas MB, Furie KL, Lev MH, Ay H, Singhal AB, Greer DM, Harris GJ, Halpern E, Koroshetz WJ, Smith WS: National Institutes of Health Stroke Scale score is poorly predictive of proximal occlusion in acute cerebral ischemia. Stroke 2009; 40:2988-2993.

21 Karnath HO, Fruhmann Berger M, Küker W, Rorden C: The anatomy of spatial neglect based on voxelwise statistical analysis: a study of 140 patients. Cereb Cortex 2004;14:1164-1172.

22 Strbian D, Ahmed N, Wahlgren N, Kaste M, Tatlisumak T; SITS Investigators: Intravenous thrombolysis in ischemic stroke patients with isolated homonymous hemianopia: analysis of Safe Implementation of Thrombolysis in Stroke-International Stroke Thrombolysis Register (SITS-ISTR). Stroke 2012;43:2695-2698. 